\title{
Pronóstico reproductivo posterior a un embarazo ectópico según modalidad de tratamiento
}

\author{
María Carolina Silva $G_{j}{ }^{1}$, Joaquín Errázuriz $V_{j}^{1}$, María José Urzúa $V^{1}{ }^{1}$, Francisco Sumar U. ${ }^{a}$, \\ Fernando Troncoso $R{ }^{1}$, Carlos Rondini F-D. ${ }^{1}$, Alvaro Insunza $F^{1}$ \\ 1 Unidad de Gestión Clínica de la Mujer y el Recién Nacido, Hospital Padre Hurtado, Facultad de Medicina Clínica \\ Alemana-Universidad del Desarrollo, Santiago de Chile.
}

a Interno, Escuela de Medicina, Clínica Alemana- Universidad del Desarrollo. Santiago de Chile.

\section{RESUMEN}

Introducción: Entre un 20 - 60\% de las pacientes que presentan un embarazo ectópico (EE) desarrollarán infertilidad en el futuro. Objetivo: Comparar las tasas de fertilidad posterior a un EE según tipo de tratamiento: médico con Metotrexato o quirúrgico, en un grupo de pacientes sin acceso a técnicas de fertilización asistida. Métodos: Presentamos un estudio de cohorte retrospectivo de pacientes con diagnóstico de EE tratadas en el Servicio de Ginecología del Hospital Padre Hurtado, entre enero de 2002 y diciembre de 2007. Se excluyeron pacientes con EE cervical o cornual, las de manejo expectante, y pacientes esterilizadas. El seguimiento mínimo fue de 4 años posterior al EE. Resultados: Se diagnosticaron $288 \mathrm{EE}$. Se excluyeron del estudio 69 pacientes. De los 219 casos restantes, se obtuvo seguimiento completo en 193 casos $(88,1 \%)$. De los 193 casos analizados, 128 fueron pacientes sometidas a resolución quirúrgica, consistente en una salpingectomía de la tuba comprometida y 65 pacientes se trataron con Metotrexato. En 18 de éstas últimas, fracasó el tratamiento médico, requiriendo de una salpingectomía diferida. Excluyendo a estas 18 pacientes, la tasa de embarazo para el grupo de resolución quirúrgica fue de $83,6 \%$ (107 de 128 pacientes) y para el grupo de manejo médico con Metotrexato fue de $80,9 \%$ (38 de 47 pacientes), sin diferencia estadísticamente significativa entre ambos grupos $(p=0,67)$. Conclusión: En este estudio, las tasas de embarazo para ambos grupos de manejo fueron similares, siendo mayores que las reportadas por la literatura.

\section{PALABRAS CLAVE: Embarazo ectópico, pronóstico reproductivo, metotrexato}

\section{SUMMARY}

Introduction: It is estimated that $20-60 \%$ of patients with ectopic pregnancy, will develop infertility on the future, but knowledge about this matter is scarce. Aims: To compare fertility rates after ectopic pregnancy, among medical treatment with methotrexate and surgery, in patients without opportunity for assisted reproductive techniques. Methods: Retrospective cohort study of patients diagnosed with ectopic pregnancy, treated in Hospital Padre Hurtado's gynecology service, Santiago - Chile, between January 2002 and December 2007. Patients with cervical or cornual ectopic pregnancy, expectant management, or patients, who didn't want future pregnancies, were excluded from the analysis. Follow up was for at least 4 years. Results: 288 ectopic pregnancies were diagnosed; 69 patients were excluded. From the 219 participants, 193 cases $(88.1 \%)$ with complete follow, were included for analysis. Surgical management, which consisted of salpingectomy of the compromised tube, was done on 128 patients. Medical management was done on 65 
patients, but 18 patients required differed salpingectomy for treatment failure. Excluding those 18 patients, the pregnancy rates for the surgical management group was of $83.6 \%(107 / 128)$ and $80.9 \%(38 / 47)$ for the medical management group, not statistically significant $(p=0.67)$. Conclusion: There are similar pregnancy rates for surgical and medical management for patients with ectopic pregnancy history; although these were higher than those reported in literature.

\section{KEY WORDS: Ectopic pregnancy, reproductive prognosis, methotrexate}

\section{INTRODUCCIÓN}

El embarazo ectópico (EE) se produce cuando el blastocisto se implanta en un sitio distinto al endometrio de la cavidad uterina. El sitio más frecuente de implantación es en las tubas uterinas, lo que ocurre en el $98 \%$ de los casos (1). A pesar de que en los últimos 20 años se han logrado grandes avances en los métodos diagnósticos y terapéuticos, el EE sigue siendo un problema de mortalidad materna, representando el $10 \%$ de ésta (2).

La incidencia, por otra parte, ha ido en aumento desde mediados del siglo XX, alcanzando a principios de los años 90 una cifra aproximada de 20 por 1000 embarazos (3). Este incremento se ha asociado al aumento de la incidencia de enfermedad inflamatoria pélvica entre los factores de mayor riesgo (4). Estudios nacionales reportan factores de riesgo similares a los de la población extranjera, entre ellos: consumo de alcohol, antecedente de cirugía ginecológica y abdominal previa, antecedente de infertilidad, haber tenido más de 2 parejas sexuales y uso de dispositivo intrauterino al momento de la concepción (5).

El cuadro clínico del EE suele manifestarse entre las 6 y 8 semanas después de la fecha de la última menstruación, y se caracteriza típicamente por la presencia de dolor en hipogastrio (99\%), amenorrea $(74 \%)$ y genitorragia $(56 \%)$, independiente de si el EE está roto o no (6), siendo la tasa de ésta complicación de un 18\% (7).

La disponibilidad de métodos de alta sensibilidad de detección de gonadotrofinas y el uso de ultrasonografía transvaginal de alta resolución han permitido realizar el diagnóstico más temprano del EE, posibilitando el uso de tratamientos menos agresivos como el uso de Metotrexato parenteral, con la intención de evitar el tratamiento quirúrgico, preservando la trompa comprometida, y eventualmente mejorando las expectativas de fertilidad futura (8).

No obstante lo anterior, se estima que la infertilidad posterior a un EE podría alcanzar cifras de un 20 a un $60 \%$ (9). Es así como la fertilidad de las pacientes posterior al EE, representa aún una interrogante, con escasos estudios de cohorte retrospectivos disponibles a la fecha que reportan tasas de embarazo en rango variable y una alta tasa de pérdida de seguimiento. Un estudio observacional de cohorte, de la población local, permitiría orientar esta interrogante. Debemos destacar que nuestra población tiene escasa o nula posibilidad de optar a técnicas de reproducción asistida, por lo cual ofrece la posibilidad de estudiar la tasa de embarazo espontáneo posterior al EE.

El objetivo primario de este estudio es comparar las tasas de fertilidad posterior a un EE según tipo de tratamiento, médico con Metotrexato o quirúrgi$\mathrm{co}$, en pacientes sin acceso a técnicas de fertilización asistida.

\section{PACIENTES Y MÉTODOS}

Se realizó un estudio de cohorte retrospectivo de las pacientes con diagnóstico de EE tratadas en la Unidad de Gestión Clínica de Mujer y el Recién Nacido del Hospital Padre Hurtado, entre los meses de enero del año 2002 y diciembre del año 2007. Los criterios de exclusión fueron aquellas pacientes con EE cervical, cornual, las de manejo expectante, así como también aquellas sin deseo de paridad futura. Los criterios de selección de tratamiento se basaron en las Guías Clínicas del Servicio de Ginecología del Hospital Padre Hurtado. El manejo médico con Metotrexato fue de elección para aquellas pacientes que se encontraban hemodinamicamente estables, sin abdomen agudo ni sospecha de embarazo ectópico roto, con subunidad B-HCG plasmática menor a $10.000 \mathrm{UI} / \mathrm{L}$, hemograma, creatininemia y pruebas hepáticas dentro de rango normal. La dosis utilizada fue $50 \mathrm{mg}$ por metro cuadrado de superficie, administrada por vía intramuscular. El seguimiento posterior de estas pacientes, se realizó de manera ambulatoria, quedando citadas para una nueva medición de subunidad B-HCG al cuarto y séptimo día desde la dosis inicial. Una segunda dosis de Metotrexato se indicó toda vez que la subunidad B-HCG plasmática al séptimo día 
no hubiese descendido al menos un $15 \%$ del valor de la medición al cuarto día.

El manejo quirúrgico, consistente en una salpingectomía de la tuba comprometida en el $100 \%$ de los casos, por vía laparoscópica o abierta, fue indicado en presencia de abdomen agudo, inestabilidad hemodinámica y frente a cualquier contraindicación de manejo médico con Metotrexato.

El seguimiento de las pacientes fue de al menos 4 años posterior al evento. La recopilación de los datos se obtuvo mediante el acceso a las fichas clínicas del archivo del hospital, el consultorio de referencia, el contacto telefónico con las pacientes y en última instancia se recurrió a la base de datos del Registro Civil e Identificación para el caso de las pacientes con pérdida de seguimiento con las fuentes previamente mencionadas. Se extrajeron datos como la edad de la paciente al momento del EE, paridad, antecedente de EE previo, cirugías abdominales, uso y tipo de método anticonceptivo utilizado, hábito tabáquico, tipo de manejo del EE, ocurrencia e intervalo de tiempo ocurrido hasta el primer evento gestacional posterior al EE y si éste culminó con un recién nacido en los brazos, aborto o recurrencia de EE. El análisis de datos se realizó con el programa SPSS 17.0. El análisis de variables continuas cuantitativas se realizó con la prueba $\mathrm{T}$ de Student mientras que para las variables categóricas se utilizó la prueba de Chi Cuadrado o Test de Fisher. Las tasas de embarazo para cada grupo de manejo se expresan a los 2 y 4 años de seguimiento. Se consideró estadísticamente significativo un $p \leq 0,05$.

\section{RESULTADOS}

Se diagnosticaron 288 pacientes con EE en el periodo de estudio. Se excluyeron del estudio 69 pacientes: 2 EE cervicales, 2 EE cornuales, 14 pacientes que se manejaron de manera expectante y 51 pacientes sin deseo de paridad futura. De los 219 casos restantes, se obtuvo seguimiento completo en 193 casos $(88,1 \%)$, los cuales fueron incluidos en el análisis. De las 26 pacientes en que hubo pérdida de seguimiento, 14 pacientes fueron de resolución quirúrgica y 12 de resolución médica y si bien el Registro Civil e Identificación no registra recién nacidos para estas pacientes con fecha posterior a la ocurrencia del EE, estas pacientes no fueron incluidas en el análisis porque se desconoce si presentaron eventos gestacionales que culminaron en aborto, recurrencia del EE o si estuvieron expuestas a embarazo durante el periodo de seguimiento.

De los 193 casos analizados, 128 fueron pacientes sometidas a resolución quirúrgica, salpingectomía abierta o laparoscópica en el $100 \%$ de las pacientes y 65 pacientes se trataron con Metotrexato. De éstas últimas, fracasaron a tratamiento médico con Metotrexato, requiriendo de salpingectomía en un intervalo variable de tiempo desde la administración de Metotrexato, corresponden a 18 casos que representan el $27,4 \%$ del grupo (Figura 1). Dado que este subgrupo de pacientes fue sometida a doble intervención, administración de Metotrexato y salpingectomía posterior, se realizó un doble análisis en la comparación de tasas de embarazo posterior al EE por grupos de manejo, uno con intención de tratar y el otro, sin intención de tratar, es decir excluyendo a las 18 pacientes que fracasaron al tratamiento médico.

Las características demográficas de ambos grupos se resumen en la Tabla I. Ambos grupos fueron comparables en cuanto a la edad, índice de masa corporal, porcentaje de pacientes primigestas, antecedente de cirugía abdominal y hábito tabáquico. Las pacientes del grupo de resolución quirúrgica reportaron un mayor uso de método anticonceptivo al momento de producirse el EE $(p=0,016)$, siendo éste el dispositivo intrauterino en una proporción significativamente mayor que en el grupo de manejo médico $(p=0,04)$. Entre los antecedentes obstétricos destaca que las pacientes sometidas a tratamiento médico con Metotrexato tenían significativamente mayor antecedente de EE previo $(p=0,012)$.

En el análisis con intención de tratar, la tasa de embarazo para el grupo de resolución quirúrgica fue de un $83,6 \%$ (107 de 128 pacientes) y para el grupo de manejo médico con Metotrexato fue de un $72,3 \%$ (47 de 65 pacientes), sin diferencia estadísticamente significativa entre ambos grupos $(p=0,065)$. El tiempo de seguimiento promedio para obtener estos resultados fue de 79,24 $\pm 20,89$ meses para el grupo de resolución quirúrgica y de $78,45 \pm 22,49$ meses para el grupo de resolución médica $(p=0,837)$. El evento gestacional culminó con un recién nacido vivo en los brazos en el $82,2 \%$ y $72,3 \%$ de los casos, respectivamente $(p=0,165)$. La tasa de recurrencia de EE fue de 9,4\% para el grupo de resolución quirúrgica y de $12,3 \%$ para el grupo de manejo médico $(p=0,528)$ (Tabla II). 


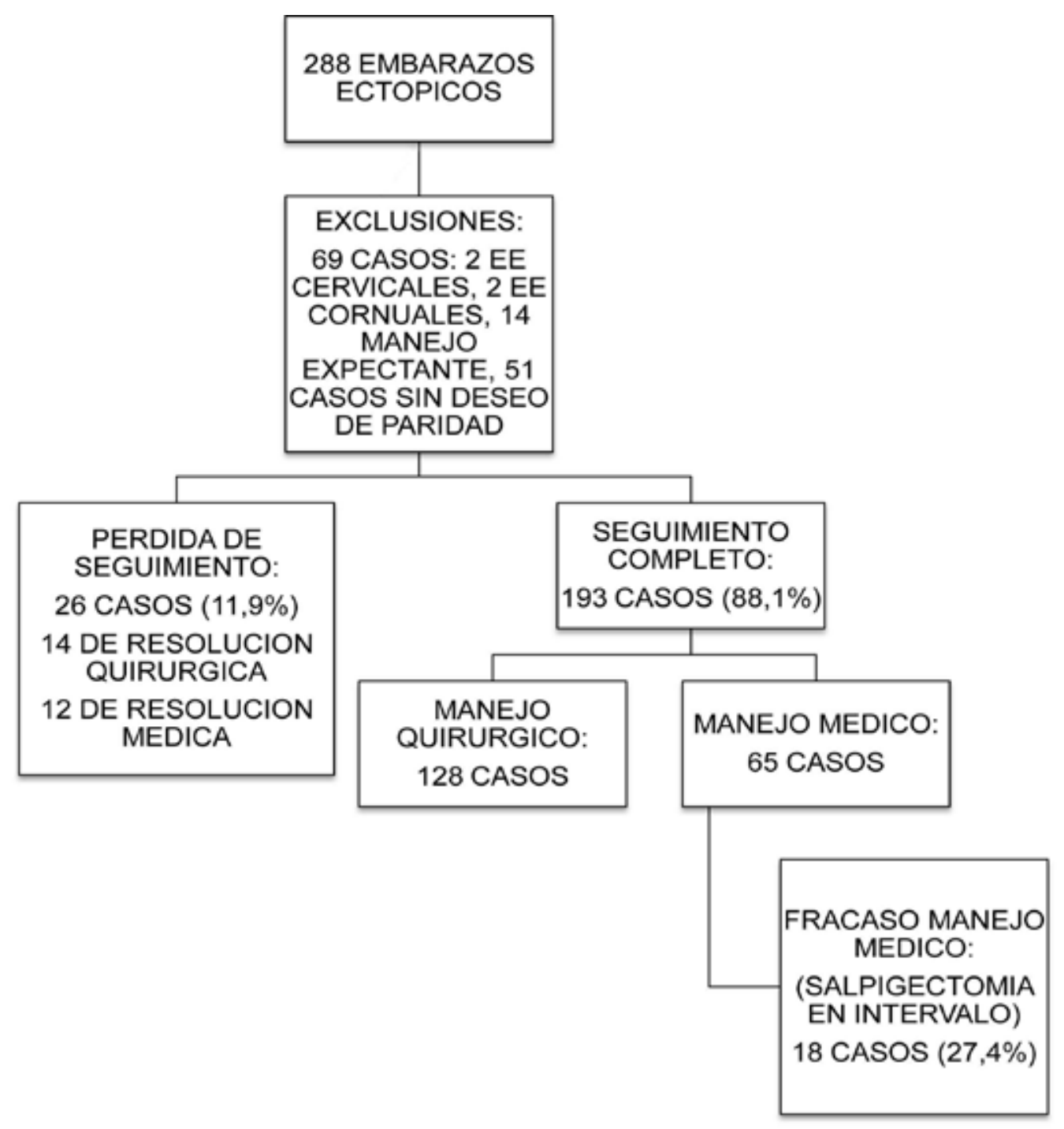

Figura 1. Algoritmo de estudio. 
Tabla I

CARACTERÍSTICAS DEMOGRÁFICAS POR GRUPO DE MANEJO

\begin{tabular}{lccc}
\hline & $\begin{array}{c}\text { Grupo } \\
\text { Salpingectomía } \\
(\mathrm{n:} \mathrm{128)}\end{array}$ & $\begin{array}{c}\text { Grupo } \\
\text { Metotrexato } \\
\text { (n: 65) }\end{array}$ & Valor p \\
\hline Edad \pm DE & $28,7 \pm 5,7$ & $27,5 \pm 5,9$ & 0,172 \\
IMC \pm DE & $26,4 \pm 4,3$ & $26,4 \pm 5,0$ & 0,965 \\
Primigestas (\% / n) & $14,1 / 18$ & $23,1 / 15$ & 0,116 \\
Usuarias MAC (\% / n) & $42,2 / 54$ & $24,6 / 16$ & $0,016^{*}$ \\
Usuarias DIU (\% / n) & $30,5 / 39$ & $13,8 / 9$ & $0,04^{\star}$ \\
Tabaquismo (\% / n) & $25,8 / 33$ & $24,6 / 16$ & 0,86 \\
Antecedentes de cirugía abdominal (\% / n) & $27,3 / 35$ & $40,0 / 26$ & 0,074 \\
Antecedentes de EE (\% / n) & $3,9 / 5$ & $13,8 / 9$ & $0,012^{*}$ \\
\hline
\end{tabular}

*Valor p estadísticamente significativo. EE: embarazo ectópico.

Tabla II

ANÁLISIS CON INTENCIÓN DE TRATAR. TASAS DE EMBARAZO, RECIÉN NACIDO VIVO Y RECURRENCIA DE EMBARAZO ECTÓPICO SEGÚN GRUPO DE MANEJO

\begin{tabular}{lccc}
\hline Análisis con intención de tratar & $\begin{array}{c}\text { Grupo } \\
\text { Salpingectomía } \\
(\mathrm{n}: 128)\end{array}$ & $\begin{array}{c}\text { Grupo } \\
\text { Metotrexato } \\
\text { (n: 65) }\end{array}$ & Valor $\mathrm{p}$ \\
\hline Tasa de embarazo $(\% / \mathrm{n})$ & $83,6 / 107$ & $72,3 / 47$ & 0,065 \\
Recien nacido vivo $(\% / \mathrm{n})$ & $82,2 / 88$ & $72,3 / 34$ & 0,165 \\
Recurrencia de embarazo ectópico $(\% / \mathrm{n})$ & $9,4 / 12$ & $12,3 / 8$ & 0,528
\end{tabular}

Dado que el seguimiento mínimo de las pacientes fue de 4 años, se compararon las tasas de embarazo por grupo de manejo, que ocurrieron en los primeros 24 meses y desde los 25 a los 48 meses de seguimiento. Del grupo de pacientes de manejo quirúrgico, el 94,3\% (101 de 107 pacientes) se embarazó en los primeros 4 años de seguimiento. En el intervalo de los primeros 24 meses se embarazó el 82,2\% (83 de 101 pacientes) y entre los 25 y 48 meses se embarazó el 17,8\% restante (18 de 101 pacientes). Por su parte, del grupo de pacientes manejadas con Metotrexato, el 97,8\% (46 de 47 pacientes) se embarazó dentro de los primeros 4 años de seguimiento, ocurriendo el 78,3\% (36 de
46 pacientes) en los primeros 24 meses y el $21,7 \%$ (10 de 46 pacientes) entre los 25 y 48 meses de seguimiento. El análisis comparativo de las tasas de embarazo en dichos intervalos de tiempo por grupo de manejo fue sin diferencia estadísticamente significativa $(p=0,575)$.

En el análisis sin intención de tratar, esto es excluyendo a las pacientes que recibieron doble intervención por fracaso al manejo médico con Metotrexato, la tasa de embarazo para el grupo de manejo médico fue de $80,9 \%$ (38 de 47 pacientes), manteniendo una diferencia estadísticamente no significativa respecto al grupo de manejo quirúrgico $(p=0,67)$ (Tabla III). 


\section{ANÁLISIS SIN INTENCIÓN DE TRATAR. TASAS DE EMBARAZO, RECIÉN NACIDO VIVO Y RECURRENCIA DE EMBARAZO ECTÓPICO SEGÚN GRUPO DE MANEJO}

\begin{tabular}{lccc}
\hline Análisis sin intención de tratar & $\begin{array}{c}\text { Grupo } \\
\text { Salpingectomía } \\
(\mathrm{n}: 128)\end{array}$ & $\begin{array}{c}\text { Grupo } \\
\text { Metotrexato } \\
(\mathrm{n}: 47)\end{array}$ & Valor $\mathrm{p}$ \\
\hline Tasa de embarazo $(\% / \mathrm{n})$ & $83,6 / 107$ & $80,9 / 38$ & 0,670 \\
Recién nacido vivo $(\% / \mathrm{n})$ & $82,2 / 88$ & $73,7 / 28$ & 0,257 \\
Recurrencia de embarazo ectópico $(\% / \mathrm{n})$ & $9,4 / 12$ & $10,6 / 5$ & 0,803 \\
\hline
\end{tabular}

Por último, la tasa de embarazo en el subgrupo de pacientes que fracasaron al manejo médico con Metotrexato y se les realizó una salpingectomía diferida por abdomen agudo, fue de un 50\% (9 de 18 pacientes) dentro de un seguimiento promedio de $75,56 \pm 19,0$ meses.

\section{DISCUSIÓN}

El objetivo del estudio fue comparar el pronóstico reproductivo posterior a presentar un $\mathrm{EE}$, según tipo de tratamiento: médico con Metotrexato o quirúrgico. Como se expuso en los resultados, el tipo de tratamiento, quirúrgico o médico con Metotrexato, no determinó un pronóstico de fertilidad significativamente mejor para una u otra modalidad, independiente de si el análisis se realiza con o sin intención de tratar ( $p=0,065$ y 0,670 respectivamente). Por otra parte, es relevante destacar que al realizar el análisis sin intención de tratar, las tasas de embarazo se equipararon notablemente entre ambos grupos de manejo, es decir la menor pero no significativa tasa de embarazo del grupo de manejo médico en el análisis con intención de tratar, se debió a que las pacientes que fracasaron al manejo médico y recibieron doble intervención, reportaron una menor tasa de embarazo (50\%, 9 de 18 pacientes) y por lo tanto representan un subgrupo de pacientes con peor pronóstico reproductivo en el seguimiento a largo plazo. Ante este hallazgo, se debe poner especial énfasis en el modo de seleccionar a las pacientes candidatas a manejo médico, dado que de fracasar la terapia, podríamos estar disminuyendo el potencial reproductivo de estas pacientes.

Son destacables las altas tasas de embarazo para ambos grupos de manejo (83 y $80 \%$ para pacientes de manejo quirúrgico y médico respectivamente), ambas mayores a las reportadas por la literatura. Previamente, diversos estudios habían intentado aproximarse en describir el potencial reproductivo de pacientes posterior a un embarazo ectópico.

En 1999, Tolaimat y cols, evaluaron la permeabilidad tubaria con histerosalpingografía en este grupo de pacientes, la cual fue de un $72 \%$ (8 de 11 pacientes) para pacientes sometidas a salpingostomía y de un $81 \%$ (9 de 11 pacientes) para pacientes tratadas con Metotrexato (10). Estas altas tasas de permeabilidad tubaria no se reprodujeron en términos de tasas de embarazo en estudios de cohorte publicados a la fecha. Kouam y cols, reportan una serie de 52 casos de embarazos ectópicos manejados quirúrgicamente, entre los cuales, la tasa acumulativa de embarazo a 36 meses fue de $32,7 \%$ (17casos) (11). Estudios de cohorte más recientes, como el de Musa y cols, reportó un $40,6 \%$ de embarazo al año de seguimiento, en una cohorte de 64 pacientes sometidas a manejo quirúrgico del embarazo ectópico (12). En el medio nacional, Troncoso y cols, en el año 2002, reportan una tasa acumulativa de embarazo intrauterino posterior al manejo médico con Metotrexato de un $57 \%$ a los 24 meses de seguimiento en una cohorte de 16 pacientes (13).

El estudio de cohorte con mayor casuística a la fecha y el más reciente, es el de Turan, quien reporta una cohorte de 133 mujeres entre los 18 y 28 años de edad, seguidas por 24 meses, dando cuenta de una tasa de embarazo de $79 \%$ (23 de 29 pacientes) para el grupo de manejo médico. Para el grupo de manejo quirúrgico, reporta una tasa de embarazo intrauterino de 65\% (35 de 55 pacientes) para las pacientes sometidas a salpingectomía y de $60 \%$ (21 de 35 pacientes) para pacientes sometidas a salpingostomía, no encontrando diferencias estadísticamente significativas entre los tres grupos en las tasas de embarazo. Este estudio, no obstante reporta tasas de embarazo elevadas, debemos considerar que corresponde a una población seleccionada de mujeres de entre 18 y 28 años de edad, por lo cual tendrían a favor un mejor pronóstico reproductivo (14). 
Entre las fortalezas del presente estudio, se destaca por una parte, el largo periodo de seguimiento, lo cual pudiera explicar las altas tasas de embarazo, dado que si bien la mayoría de los embarazos se produce dentro de los dos primeros años, hay un porcentaje no despreciable de embarazo que ocurren posterior a este intervalo sin intervención alguna. Por otra parte hay un bajo porcentaje de pacientes excluidas por pérdida de seguimiento lo que hace que los resultados sean de una precisión que permite aplicarlos a la población general.

\section{CONCLUSIÓN}

En estudios de cohorte como el actual, frecuentemente habrá pacientes que se perderán en el seguimiento. Un estudio prospectivo randomizado de las pacientes con embarazo ectópico, respondería en forma fidedigna lo que ya parece evidente en este estudio, que no habría diferencias en el pronóstico reproductivo, independiente de la modalidad de tratamiento y en el seguimiento a largo plazo, como fue en este estudio, las tasas de embarazo espontáneo son elevadas, determinado una tasa de infertilidad cercana al $20 \%$ posterior a un EE.

\section{REFERENCIAS}

1. Tulandi T. Incidence, risk factors, and pathology of ectopic pregnancy. Update; mayo 2009. Disponible en: http://www.uptodate.com.

2. Ory SJ. New options for diagnosis and treatment of ectopic pregnancy. JAMA 1992;267:534-7.

3. Centers for Disease Control and Prevention (CDC). Ectopic pregnancy--United States, 1990-1992. MMWR Morb Mortal Wkly Rep 1995;44:46-8.
4. Kamwendo F, Forslin L, Bodin L, Danielsson D. Epidemiology of ectopic pregnancy during a 28 year period and the role of pelvic inflammatory disease. Sex Transm Infect 2000;76:28-32.

5. Urrutia MT, Poupin L, Alarcón P, Rodríguez M, Stuven L. Embarazo ectópico: factores de riesgo y características clínicas de la enfermedad en un grupo de mujeres chilenas. Rev Chil Obstet Ginecol 2007;3:154-9.

6. Alsuleiman SA, Grimes EM. Ectopic pregnancy: a review of 147 cases. J Reprod Med 1982;27:101-6.

7. Job-Spira N, Fernandez H, Bouyer J, Pouly JL, Germain E, Coste J. Ruptured tubal ectopic pregnancy: risk factors and reproductive outcome: results of a population-based study in France. Am J Obstet Gynecol 1999;180:938-44.

8. Makinen J. Current treatment of ectopic pregnancy. Ann Med 1999;31:197-201.

9. Ego A, Subtil D, Cosson M, Legoueff F, Houfflin-Debarge V, Querleu D. Survival analysis of fertility after ectopic pregnancy. Fertil Steril 2001;75:560-6.

10. Tolaymat LL, Brown TL, Maher JE, Horan CA, Green BA, Ripps BA. Reproductive potential after methotrexate treatment of ectopic gestation in a community hospital. J Reprod Med 1999 Apr;44:335-8.

11. Kouam L, Kamdom-Moyo J, Ngassa P, Doh AS, Salihu HM. Management of ectopic pregnancy by laparotomy and subsequent fertility. J Obstet Gynaecol 1998;18:169-70.

12. Musa J, Daru PH, Mutihir JT, Ujah IA. Ectopic pregnancy in Jos Northern Nigeria: prevalence and impact on subsequent fertility. Niger J Med 2009;18:35-8.

13. Troncoso JL, Devoto L, Santamaría R, Fuentes A. Tratamiento médico del embarazo ectópico: evaluación prospectiva de la fertilidad. Rev Chil Obstet Ginecol 2002;67:354-9.

14. Turan V. Fertility outcomes subsequent to treatment of tubal ectopic pregnancy in younger turkish women. J Pediatric Adolesc Gynecol 2010;24:251-5. 